\title{
Organ cross-sensitization mechanisms in chronic diseases related to the genitourinary tract
}

Tsuyoshi MAJIMA* and Naoto SASSA

Department of Urology, Aichi Medical University, 1-1 Yazakokarimata, Nagakute City, Aichi 480-1195, Japan

Submitted September 12, 2020; accepted in final form August 30, 2021

\begin{abstract}
There are various refractory chronic inflammatory diseases related to the genitourinary tract, such as interstitial cystitis/bladder pain syndrome and chronic prostatitis/chronic pelvic pain syndrome. It has been reported that in the general population, these diseases are related to other chronic illnesses, such as irritable bowel syndrome or vulvodynia. Herein, we review papers regarding pelvic organ cross-sensitization, a factor which is considered to contribute to these relationships. Several other researchers and ourselves have reported that noxious stimuli from a diseased pelvic organ are transmitted to an adjacent normal structure via shared sensory neural pathways at the prespinal, spinal, and supraspinal levels, resulting in functional changes in the adjacent normal structure. In conclusion, since there are few treatments to cure interstitial cystitis/bladder pain syndrome and chronic prostatitis/chronic pelvic pain syndrome completely, further studies regarding organ cross-sensitization may provide new insights into the pathophysiology and treatment strategies for these diseases.
\end{abstract}

Key words: organ cross-sensitization, interstitial cystitis, bladder pain syndrome, chronic prostatitis, chronic pelvic pain syndrome

\section{Introduction}

There are various chronic inflammatory diseases related to the genitourinary tract, such as interstitial cystitis/bladder pain syndrome (IC/BPS) and chronic prostatitis/chronic pelvic pain syndrome (CP/CPPS). Although these diseases lead to marked deterioration of quality of life (QOL) in patients due to pain or urinary urgency, the pathophysiology of these diseases remains unclear. Epidemiological studies have shown that patients with these diseases are more likely to have other chronic illnesses, such as irritable bowel syndrome (IBS) or vulvodynia, in comparison with the general population $(1,2)$. Pelvic organ cross-sensitization is con-

*The Tsuneo Shiratori Award 2019 winner. Corresponding author: Tsuyoshi Majima, Department of Urology, Aichi Medical University, 1-1, Yazakokarimata, Nagakute City, Aichi, Japan

Phone: +81-561623311 Fax: +81-561638166 E-mail: majima.tsuyoshi.888@mail.aichi-med-u.ac.jp

(C)2021 The Japan Society of Smooth Muscle Research 
sidered to be one of the factors contributing to these relationships (3). It is known that afferent neural pathways innervating pelvic organs converge in the central nervous system. Cross-sensitization implies transmission of noxious stimuli from a diseased pelvic organ to an adjacent normal structure, resulting in functional changes in the latter. Cross-sensitization in the pelvis mainly occurs via shared sensory neural pathways at the prespinal, spinal, and supraspinal levels (4). The aim of this paper is to review the pathophysiology of chronic diseases related to the genitourinary tract in terms of pelvic organ cross-sensitization.

\section{Interstitial cystitis/bladder pain syndrome}

IC/BPS is a chronic pain syndrome, the symptoms of which include urinary frequency, urinary urgency, and pain (5). According to an epidemiological study, the hazard ratio for developing IC/BPS in subjects with endometriosis compared to subjects without endometriosis was 4.43 (95\% confidence interval (CI): 2.13-9.23) (6). In addition, another study showed that patients with interstitial cystitis were more likely to be diagnosed with IBS (odds ratio (OR) 11; 95\% CI 2.7-52) (7). Several basic laboratory studies have shown that pelvic organ cross-sensitization plays a role in the clinically overlapping symptoms of these diseases. For example, it has been shown in rats that surgical induction of endometriosis significantly reduced micturition thresholds and increased bladder inflammation (8). Moreover, there have been reports showing pelvic organ cross-sensitization between experimental colitis-induced bladder overactivity and painful bladder sensations $(9,10)$. We investigated whether microglia in the spinal cord contributed to central sensitization in experimental colitis in rats (9). In our study, rats with experimental colitis showed bladder overactivity and increased pain bladder sensations. Additionally, the number of spinal microglia was significantly larger in these rats than that in the control rats. Inhibition of spinal microglia significantly recovered these changes in bladder hyperexcitability caused by experimental colitis (9). These results imply that microglia may play a role in the colon-to-bladder neural cross-talk, at the level of the spinal cord, in a rat model of colitis. Furthermore, another study indicated that experimental colitis led to bladder hypersensitivity via pelvic organ cross-sensitization between the colon and bladder, which was attributed to the activation of dichotomized afferent neurons innervating both organs, at the level of the dorsal root ganglia (DRG) (10). Thus, it may be assumed that activation of the spinal microglia and/or afferent neurons in the DRG is one of the underlying mechanisms in pelvic organ cross-sensitization between the bladder and colon or between the bladder and uterus. It is possible that these pathophysiologies could contribute to the overlapping symptoms in patients with IC/BPS.

\section{Chronic prostatitis/chronic pelvic pain syndrome}

$\mathrm{CP} / \mathrm{CPPS}$ is defined when pelvic pain is present for $\geq 3$ of the preceding 6 months, and no other identifiable causes have been detected. Other symptoms include obstructive or irritative voiding difficulties, ejaculatory pain, and sexual dysfunction. Men affected by CP/CPPS have significantly decreased QOL (11). A clinical study showed that Hunner-type IC is a common comorbidity among patients with refractory CP/CPPS (12). Several basic studies have shown that pelvic organ cross-sensitization plays a role in the clinically overlapping symptoms of these diseases $(13,14)$. A basic study in rats demonstrated that experimental prostatitis caused detrusor overactivity (13). In addition, it was reported that in rats a significant number of DRG neurons with dichotomized afferents innervate both the prostate and bladder (13). In rats with prostatic inflammation, the populations of transient receptor potential vanilloid 1, transient receptor potential ankyrin 1, and P2X2 receptors increased due to mRNA activity in the bladder afferent and double-labeled neurons compared with the non-labeled neurons (13). This study concluded that prostate-to-bladder cross-sensitization through the primary afferent pathways, which contain dichotomized afferents, could be an important mechanism contributing 
to bladder overactivity and afferent hyperexcitability induced by prostatic inflammation (13). Therefore, it is possible that pelvic organ cross-sensitization is one of the underlying pathophysiologies of $\mathrm{CP} / \mathrm{CPPS}$.

\section{Cold temperature and overactive bladder}

It is known that exposure to low temperature tends to cause lower urinary tract symptoms, such as urgency and urinary frequency in patients with overactive bladder (15). According to animal studies, crosssensitization between the skin and bladder was associated with this phenomenon $(16,17)$. Localized cooling of the skin evoked rapid bladder contractions and voids in anesthetized mice. These responses were strongly attenuated in transient receptor potential melastatin 8 (TRPM8) knockout mice (16). In addition, another study indicated that spraying TRPM8 channel agonist onto the skin induced detrusor overactivity in rats (17). In this study, the authors speculated that skin to bladder cross-sensitization through TRPM8 channels on the skin may be mediated by the nervous system, including either spinal tract neurons or via the pontine micturition center.

\section{Conclusion}

As reviewed in this paper, organ cross-sensitization could be associated with the pathophysiology of several chronic diseases related to the genitourinary tract, such as IC/BPS and CP/CPPS. Since there are few treatments to cure these diseases completely, further studies regarding organ cross-sensitization may provide new insights into the pathophysiology and treatment strategies for these diseases.

\section{Conflict of Interest}

We have no conflicts of interest to declare.

\section{References}

1. Clemens J.Q., Brown S.O., Kozloff L, Calhoun E.A. Predictors of symptom severity in patients with chronic prostatitis and interstitial cystitis. J Urol. 2006; 175(3 Pt 1): 963-6 discussion 967. [Medline] [CrossRef]

2. Fariello J.Y., Moldwin R.M. Similarities between interstitial cystitis/bladder pain syndrome and vulvodynia: implications for patient management. Transl Androl Urol. 2015; 4(6): 643-52. [Medline]

3. Pezzone M.A., Liang R, Fraser M.O. A model of neural cross-talk and irritation in the pelvis: implications for the overlap of chronic pelvic pain disorders. Gastroenterology. 2005; 128(7): 1953-64. [Medline] [CrossRef]

4. Malykhina A.P. Neural mechanisms of pelvic organ cross-sensitization. Neuroscience. 2007; 149(3): 660-72. [Medline] [CrossRef]

5. Birder L.A. Pathophysiology of interstitial cystitis. Int J Urol. 2019; 26(Suppl 1): 12-5. [Medline] [CrossRef]

6. Wu C.C., Chung S.D., Lin H.C. Endometriosis increased the risk of bladder pain syndrome/interstitial cystitis: a population-based study. Neurourol Urodyn. 2018; 37(4): 1413-8. [Medline] [CrossRef]

7. Novi J.M., Jeronis S, Srinivas S, Srinivasan R, Morgan M.A., Arya L.A. Risk of irritable bowel syndrome and depression in women with interstitial cystitis: a case-control study. J Urol. 2005; 174(3): 937-40. [Medline] [CrossRef]

8. Morrison T.C., Dmitrieva N, Winnard K.P., Berkley K.J. Opposing viscerovisceral effects of surgically 
induced endometriosis and a control abdominal surgery on the rat bladder. Fertil Steril. 2006; 86(4 Suppl): 1067-73. [Medline] [CrossRef]

9. Majima T, Funahashi Y, Kawamorita N, Takai S, Matsukawa Y, Yamamoto T, Yoshimura N, Gotoh M. Role of microglia in the spinal cord in colon-to-bladder neural crosstalk in a rat model of colitis. Neurourol Urodyn. 2018; 37(4): 1320-8. [Medline] [CrossRef]

10. Yoshikawa S, Kawamorita N, Oguchi T, Funahashi Y, Tyagi P, Chancellor M.B., Yoshimura N. Pelvic organ cross-sensitization to enhance bladder and urethral pain behaviors in rats with experimental colitis. Neuroscience. 2015; 284: 422-9. [Medline] [CrossRef]

11. Franco JVA, Turk T, Jung J.H., Xiao Y.T., Iakhno S, Tirapegui F.I., Garrote V, Vietto V. Pharmacological interventions for treating chronic prostatitis/chronic pelvic pain syndrome: a Cochrane systematic review. BJU Int. 2020; 125(4): 490-6. [Medline] [CrossRef]

12. Ueda M, Sengiku A, Kono J, Negoro H, Saito R, Yoshimura N, Ogawa O, Ueda T. Low bladder capacity is an important predictor for comorbidity of interstitial cystitis with Hunner's lesion in patients with refractory chronic prostatitis/chronic pelvic pain syndrome. Int J Urol. 2019; 26(Suppl 1): 53-6. [Medline] [CrossRef]

13. Funahashi Y, Takahashi R, Mizoguchi S, Suzuki T, Takaoka E, Ni J, Wang Z, DeFranco D. B., de Groat W. C., Tyagi P, Yoshimura N. Bladder overactivity and afferent hyperexcitability induced by prostateto-bladder cross-sensitization in rats with prostatic inflammation. J Physiol. 2019; 597(7): 2063-78. [Medline] [CrossRef]

14. Chen Y, Wu X, Liu J, Tang W, Zhao T, Zhang J. Distribution of convergent afferents innervating bladder and prostate at dorsal root Ganglia in rats. Urology. 2010; 76(3): 764.e1-6. [Medline] [CrossRef]

15. Ghei M, Malone-Lee J. Using the circumstances of symptom experience to assess the severity of urgency in the overactive bladder. J Urol. 2005; 174(3): 972-6. [Medline] [CrossRef]

16. Uvin P, Franken J, Pinto S, Rietjens R, Grammet L, Deruyver Y, Alpizar Y.A., Talavera K, Vennekens R, Everaerts W, De Ridder D, Voets T. Essential role of transient receptor potential M8 (TRPM8) in a model of acute cold-induced urinary urgency. Eur Urol. 2015; 68(4): 655-61. [Medline] [CrossRef]

17. Lei Z, Ishizuka O, Imamura T, Noguchi W, Yamagishi T, Yokoyama H, Kurizaki Y, Sudha G.S., Hosoda T, Nishizawa O, Andersson K.E. Functional roles of transient receptor potential melastatin 8 (TRPM8) channels in the cold stress-induced detrusor overactivity pathways in conscious rats. Neurourol Urodyn. 2013; 32(5): 500-4. [Medline] [CrossRef] 